\title{
SIMULATION OF A MULTI-ROBOT BASED ASSEMBLY SYSTEM
}

\author{
Lazinica, A. ${ }^{*} \&$ Kordic, V.* \\ *Vienna University of Technology, Intelligent Manufacturing Systems Group, \\ Karlsplatz 13/311, A-1040 Vienna, Austria \\ E-mail: lazinica@mail.ift.tuwien.ac.at \\ ${ }^{* *}$ Vienna University of Technology, Automation and Control Institute, \\ Gusshausstrasse 27-29, A-1040 Vienna, Austria \\ E-mail: kordic@acin.tuwien.ac.at
}

\begin{abstract}
Scope of this paper is to present current development of the Bionic Assembly System and simulation of a simplified system in Webots software. In this paper we present the hardware design and design of controller for mobile manipulator and a design of transport mobile robot behaviour. The simulation is done with all kinematical and dynamical characteristics of each component, i.e. with physics. Results presented are just a base for starting to develop a simulation of a whole Bionic Assembly System and to test the concept of a system and ideas.
\end{abstract}

Key Words: Multi-Robot System, Assembly, Self-Organisation, Simulation

\section{INTRODUCTION}

There is a widely accepted attitude that following three manufacturing paradigms can better address existing and predicted future manufacturing demands: biologically inspired manufacturing - Ueda, 1992; fractal factory - Warnecke, 1993 and Holonic Manufacturing van Brussel, 1992. Such manufacturing can deliver custom-manufactured products to an increasingly discriminating customer base [1].

This paper is a further development of Bionic Assembly System proposed in [2]. Bionic Assembly System solves following problems of existing manufacturing systems:

1. Lack of flexibility to deal with the diversification of products and their uses. Existing manufacturing systems cannot cope with changes in product design and manufacturing plans nor keep in pace with the dynamics of the market.

2. Lack of flexibility to deal with replacement of equipment and the increasing complexity of the system itself. Manufacturing equipment cannot be easily replaced nor can simultaneous use of new and old machinery be easily integrated.

3. Planning execution loop. As complexity of manufacturing systems increase time between planning and executing becomes too long. That is, we have a big chance that new plan lead only to applicable solution. In such cases it is questionable if planning/scheduling/rescheduling pay off.

Main elements of a proposed system are autonomous mobile robots. They have to function autonomously, have to adapt themselves and act in strong co-relation between each other and their environment (shop-floor). Design of task-oriented behavior of single robot and cooperation between different robot classes under production constraints is a main task to be solved. 


\section{BIONIC ASSEMBLY SYSTEM}

The concept of Bionic Assembly System was developed as an answer on a real industrial demand to significantly reduce the production costs of electrical motors in mass production with open range of variants of types of electrical motors, which is in permanent change. Such kind of production demands high level of flexibility, adaptability and intelligence of assembly system. Rash development of new forms of production/machining/assembly systems makes used equipment obsolete in a short time. Hence assembly systems have to be autonomous and modular [3].

Bionic Assembly System is composed out of two subsystems (Fig. 1): Core subsystem and Supplementary subsystem of BAS, these subsystems are divided by system border. The core subsystem is central part of assembly system. It includes: all assembly stations, mobile robots, and assembly pallets, repair station, quality control station.

The dominating activity in the core subsystem is assembly, quality control, and repair. The supplementary subsystem is surrounding the core subsystem. The main activity in this subsystem is the storage of parts and components. The main activity on the system border between the core and supplementary subsystems is the supply of the assembly stations with the parts and components, which have to be assembled. The material flow crossing system border includes additionally the set-ups for the stations. The pool of pallets is buffer storage of assembly pallets in the case that the pallet are not designed to be universal assembly pallet for all type of products. The main material output from core subsystem is in the form of finalised products with good quality. These products are leaving system at loading/unloading station and after packing they are ready to delivering to the customer. Over the same station is organised the output of bad products and half products did not complete the assembly and and/or can not be repaired.

\subsection{Mobile Robot Classes}

The state-of-the-art of mobile robot technology and predictions of future development are giving a clear view that mobile robots are going to be essential part of every manufacturing process in not so far future [4]. Robots now can intelligently go from place to place and collect parts and take them to the appropriate work cell which opens up a new different way of structuring the manufacturing environment. The main advantage of mobile robots is their flexibility. In materials handling operations, this will eliminate the element of human error, reducing goods handling accidents and losses. The point where mobile robots could be efficiently integrated into manufacturing systems is very near. Keeping in mind these facts, Bionic Assembly System is completely structured of mobile robots. To realize Bionic Assembly System six mobile robot classes are introduced [5]. All assembly units of the same type create one class, e.g. all transport mobile robots builds transport class (Table I).

Transport and assembly class builds a basis of Bionic Assembly System. They have to deal with following sources of uncertainty and dynamic events:

- Incoming parts variation: Variable availability and arrival rates and poses of incoming parts

- Variable quality of incoming parts: Faulty parts must be removed before they either cause errors in system, or become assembled into products.

- Mixed batch of parts to be assembled: The assembly station assembling a range of variants of electrical motors types.

- Variable availability of resources: Resources are tools and machines with which the assembly station needs to directly coordinate. 


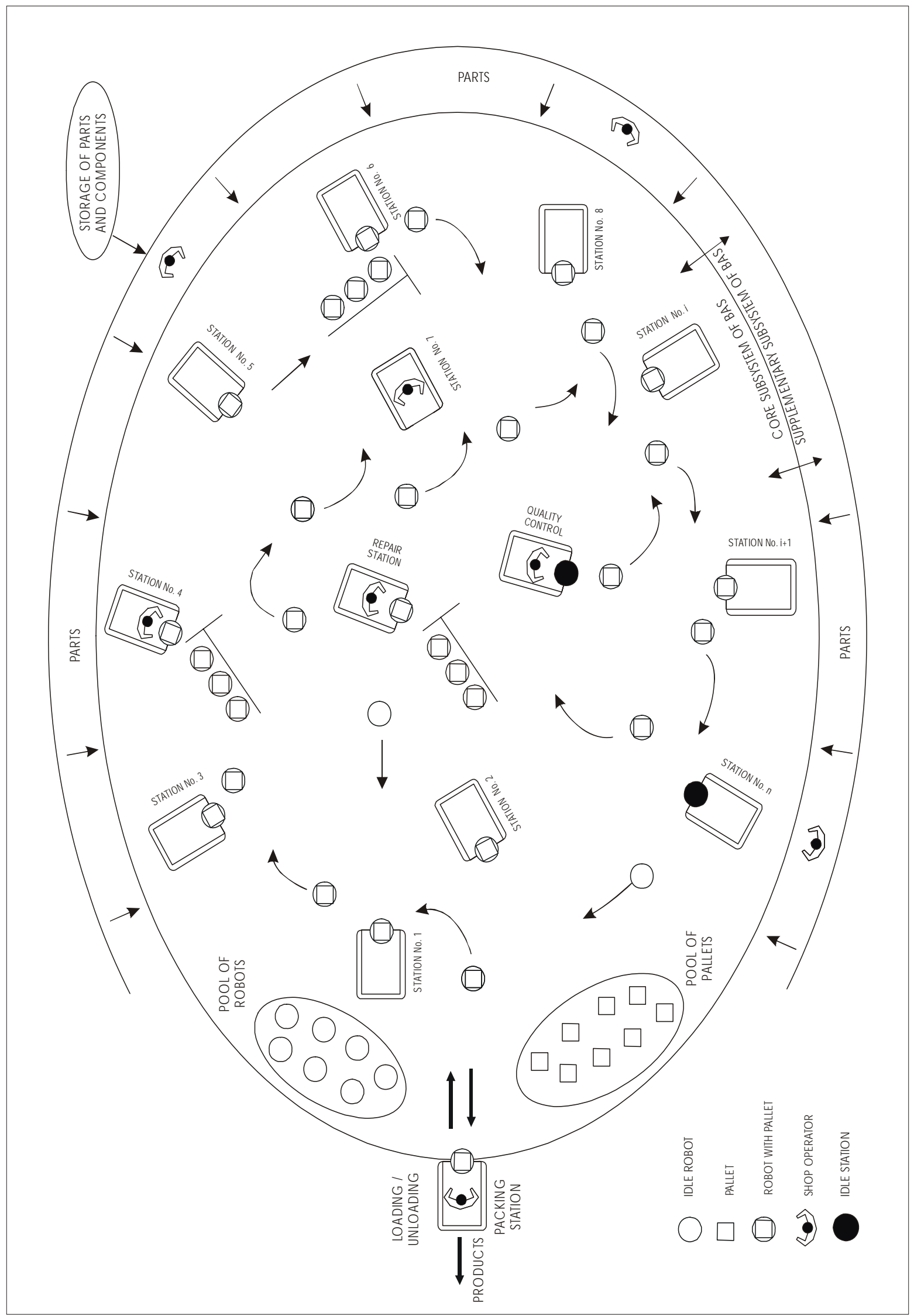

Figure 1: A concept of Bionic Assembly System. 
- Response to dynamical events in self - organising assembly system: For example opening alternative assembly ways to bypass machines break ups or allowing more assembly stations and transport agents in core subsystem to increase production capacity. Supply class provides assembly class units with needed parts, energy class exchanges empty batteries. Booth actions take place at actual position of assembly unit supporting self organised assembly structure.

Table I: Decomposition and function of classes.

\begin{tabular}{|l|l|l|}
\hline \multicolumn{1}{|c|}{ Class } & \multicolumn{1}{|c|}{ Function } & \multicolumn{1}{c|}{ Realisation } \\
\hline Transport & $\begin{array}{l}\text { Place of assembly, carrier of assembling } \\
\text { product, assembly related information carrier. }\end{array}$ & $\begin{array}{l}\text { Autonomous mobile robot with } \\
\text { exchangeable palette. }\end{array}$ \\
\hline Assembly & $\begin{array}{l}\text { Assembling of assembly steps in charge, } \\
\text { storage for corresponding assembly parts. }\end{array}$ & $\begin{array}{l}\text { Mobile robots with manipulator and } \\
\text { part storage for three different } \\
\text { assembly parts. }\end{array}$ \\
\hline Supply & $\begin{array}{l}\text { Delivering of assembly parts to assembly } \\
\text { stations. }\end{array}$ & $\begin{array}{l}\text { Autonomous mobile robots adapt to } \\
\text { transport assembly parts in quantities. }\end{array}$ \\
\hline Energy & $\begin{array}{l}\text { Changing batteries of an assembly station at } \\
\text { the actual assembly station position and } \\
\text { transporting of these to the charging station. }\end{array}$ & $\begin{array}{l}\text { Autonomous mobile robots adapt to } \\
\text { transport and exchange batteries. }\end{array}$ \\
\hline Repair & $\begin{array}{l}\text { Repairing or removing of non repairable } \\
\text { motors from assembly process. }\end{array}$ & Fix box. Shop floor operator driven. \\
\hline Unload & $\begin{array}{l}\text { Taking over of finalised products with good } \\
\text { quality. }\end{array}$ & Fix mounted industrial robot. \\
\hline
\end{tabular}

\subsection{Descriptive Algorithm of Robot - Station Communication and elements of working scenario}

The basic algorithm of the communication between active robot agent and stations is the key part of working scenario:

Start

\{

Robot state after last operation \{ Type of Assembly

Pallet, Status of Pallet, Type of Product, Next Step of Assembly, Quality State of Product\}

if $\{$ Next step of Assembly is Packing\}

then $\{$ Robot, new assembly order has to go to

the Loading/Unloading Station\}

if $\{$ Quality State of Product is negative $\}$

then $\{$ Robot has to go the Repair Station.

Wait to the shop floor operator. Shop floor operator will try to repair the product if this is not possible he will remove it from the system, and will prepare the pallet and mobile robot for the assembling of next (new) product. Results of repair operation: State of Assembly and Quality State\}

if \{Station become active or pasive $\}$

then Rearrangement of the Queues of

alternative Stations\}

if $\{$ Quality State of Product is positive and 


\section{Next Operation is Assembly Operation}

then \{Find out which Station(s) can perform next Assembly Operation; If there are more than one find out which is better, taking into the consideration existing queues and priorities\}

if $\{$ Mobile robot is present and Station is busy or

there are waiting robot(s) with equal or higher priority or there are Robot(s) of equal priority which are waiting for longer time\}

then Mobile robot has to wait in the queue

of the Station for next operation\}

if $\{$ Station is free and there are no robot(s) with higher priority in the queue\}

then $\{$ Docking, Execute Assembly Operation,

Check the Quality of Results of Assembly Operation, Write new State of Assembly and Quality State of Product, Undocking\}

Robot state after last operation

\}

End

\section{SIMULATION OF BAS IN WEBOTS}

We have decided to develop a simulation of Bionic Assembly System firstly and then to transfer the controllers on real, physical robots. Simulation is cost and time effective, and today's market offers realistic simulators in which robots behaviors are $90 \%$ the same as in the real, physical world. Of course, you can never compare the work with real and simulated robots, but our goal is to research the system as a whole and not a single robot as a unit. The Webots professional mobile robot simulation software seemed as a perfect choice. The software is described and presented in later chapter.

The simplified version of Bionic Assembly System that we have developed in Webots is shown on Fig. 2.

\subsection{Transport Mobile Robot}

The function of transport mobile robot is to carry a palette on which the product is to be assembled and to "circle" from one assembly station to another [6]. He has to be capable of avoiding static (assembly stations, machine parts ...) and moving (other transport robots) obstacles.

At the beginning transport mobile robot should get an order which kind of product should be assembled. The products are represented by three cubes which are in three different colors: red, green and blue. Different combinations of these colors represent different kind of products. When the transport mobile robot has the type of the product it has to go to the loading station to get one palette on which the product should be assembled. Then it has to go from one assembly station to another in order to assembly the right combination of the cubes. After the product is assembled it has to go to the unloading station which takes the palette with final product. At this moment, transport mobile robot has fulfilled his assembly mission and it is going back to the initial position (robot pool) and waits for new order to come. Since the robot is equipped with battery sensor which measures the state of the energy level, robot should go to the recharging station first if its battery level is less then $15 \%$ of full energy. By this stage one work cycle of a transport mobile robot is finished. 
At this moment we have developed the controller which navigates the transport mobile robot from the beginning to the end of the cycle. The controller is written in $\mathrm{C}++$ and vector field histogram field method has been used for navigation [7, 8]. It is presented in Fig. 3.

The main function looks as follows:
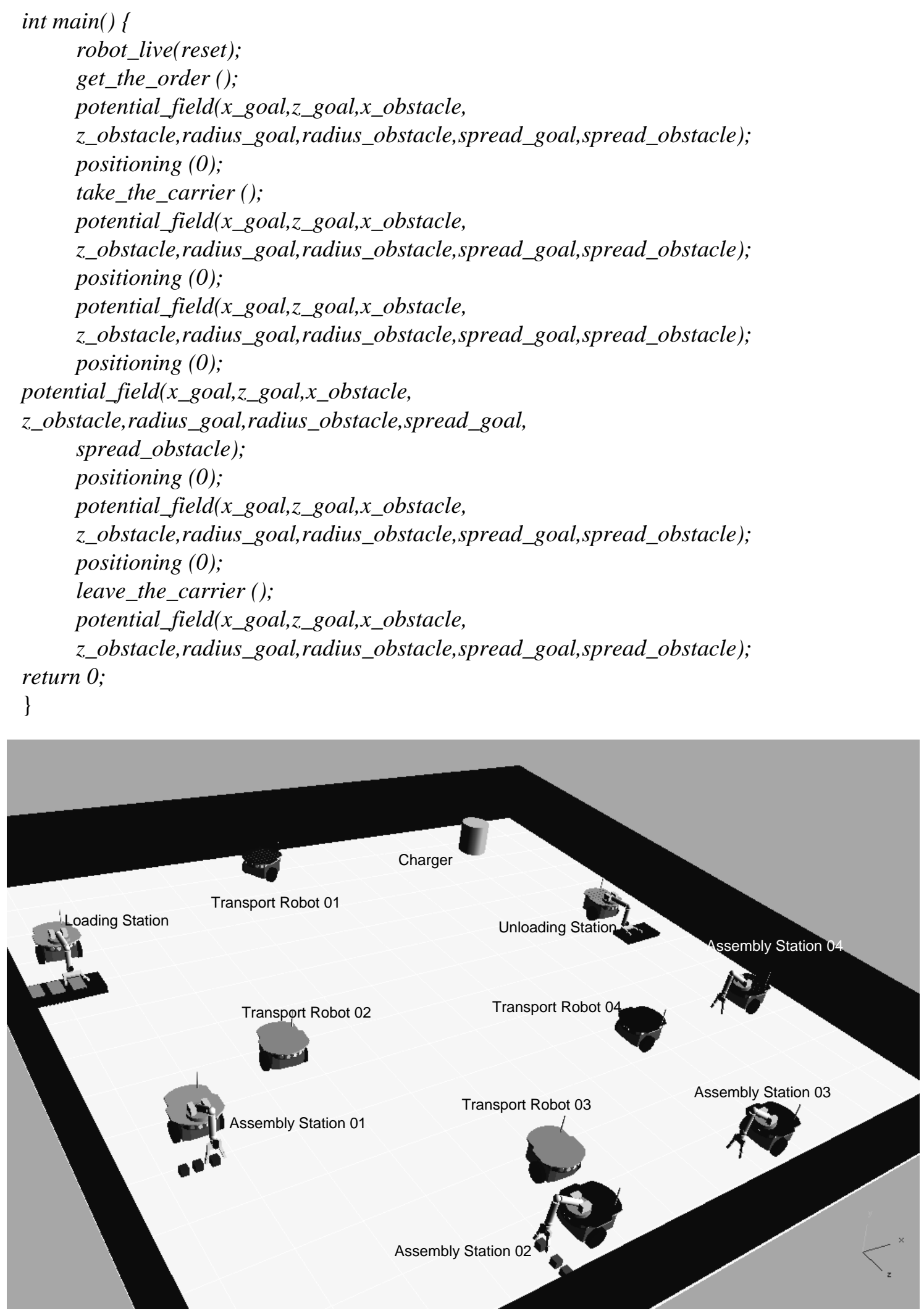

Figure 2: Bionic Assembly System in Webots Simulator. 
The functions are described as follows:

- robot_live - this function starts the robot and all its components,

- get_the_order - with this function a menu in windows is opened where we should input the desired colour combination of cubes to be assembled,

- take_the_carrier - robot is at the palette storage and takes the empty palette,

- potential_field - this function calls the potential field method and it navigates the robot through the environment,

- positioning - this function is used to position the robot in the correct angle on the goal position,

- leave_the_carrier - robot is at the storage of final products and leaves the palette.

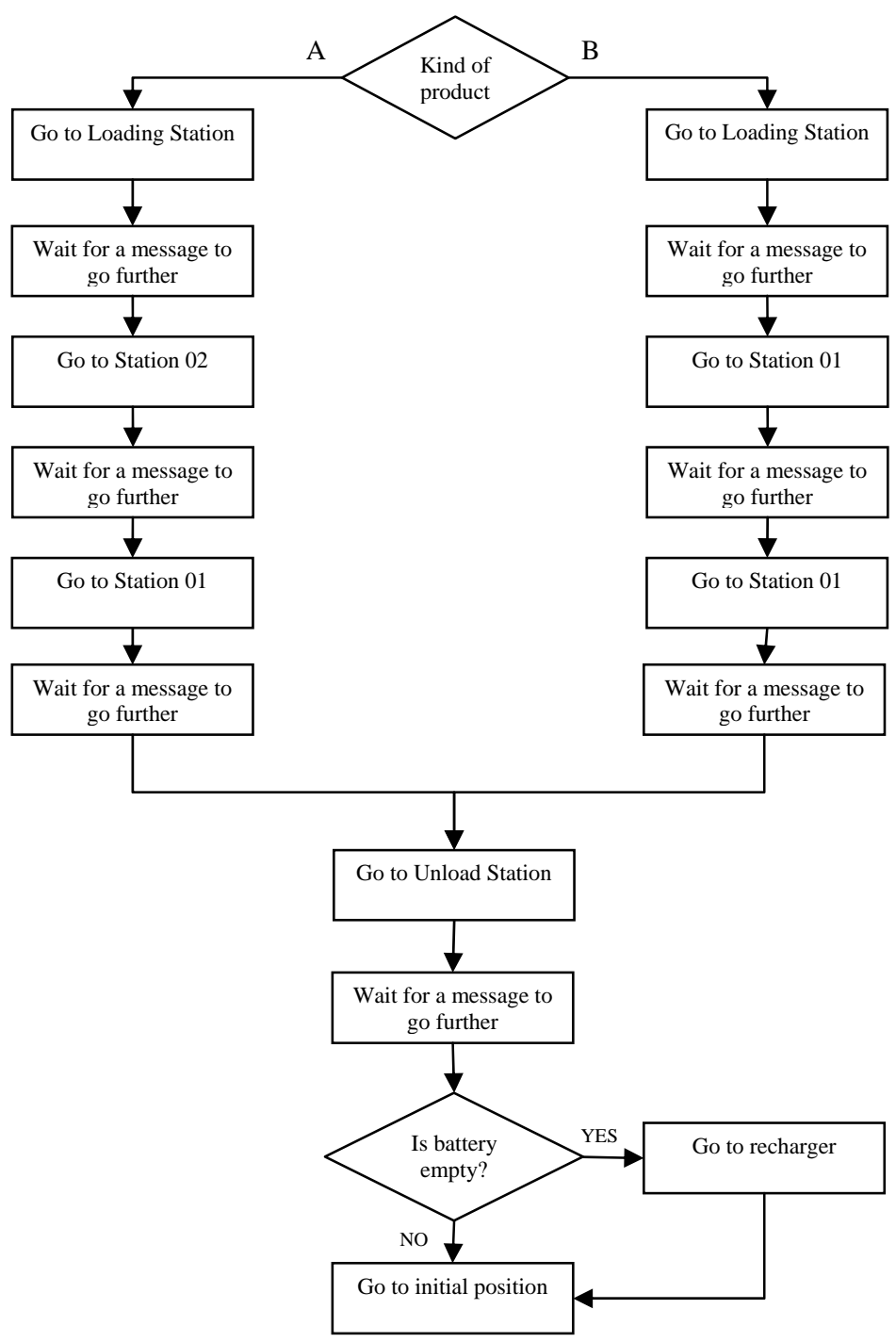

Figure 3: Transport Mobile Robot Controller.

\subsection{Assembly Station Robot}

Two most important robot classes in Bionic Assembly System are Assembly Station Robot and Transport Mobile Robot. As already mentioned before the function of assembly station is the manipulation of assembly parts - positioning of an assembly part on a palette which is 
situated on a transport mobile robot. Assembly station is composed of industrial robot, conveyors, assembly parts and vision system. In this simplified version we only use mobile robot arm.

\section{- Assembly Station Controller}

We have developed a behavior-based controller for the assembly station. The robot cycle begins when he receive a message from a transport robot to start the assembly process. The robot is taking a part from a ground, lift the part up and then turn and in that way position itself in a right position. Then robot stops, put the part on a palette, lifts the arm and turn itself to initial position. Robot cycle is finished and it is sending a message to a transport mobile robot to go further.

The basic behaviours of the assembly station robot are:

1.) Grasp the assembly part - robot arm grasp the part with a fingers. Every finger has distance sensor which measures the distance between the finger and the object and in that way we could stop the finger servo movements when the object is grasped.

2.) Lift the arm up - robot is lifting his arm up with the arm servo motors.

3.) Positioning - robot has to turn itself in a place in order to position properly in relation to transport mobile robot. We gave the same speed to both robot motors, but with different orientation. In every robot step we are measuring the robot orientation angle and when the angle is the same as the goal position angle (alpha) robot stops.

4.) Put the arm down - robot is putting the arm down in order to leave the assembly part. The position of robot arm is not always the same, since it depends from the assembly step. At the beginning of the cycle, robot received the message from transport mobile robot with his ID and assembly step. In that way he knows what is a position of the assembly part.

5.) Leave the assembly part - robot is leaving the assembly part on the assembly palette.

6.) Communication - robot has to be able to communicate with a transport mobile robots. This communication is realised through radio connection. It is equipped with emitter and receiver devices and capable of sending and receiving the messages. At the beginning robot receives the message from a transport mobile robot. The message is composed of two variables. The first one is describing the transport robot ID and the second one assembly step being performed. In that way assembly station knows exactly on which robot it has to put the assembly part and in which position. At the end of the cycle assembly station is sending a message to the transport mobile robot (that is a reason why station needs transport robot's ID) that the assembly step is finished and that it can go further (to the next assembly station).

The block diagram of a controller is shown on Fig. 4. The controllers are written in C++ and the robot behaviour simulated in Webots simulator.

\section{- Modeling of Assembly Station Robot in Webots Simulation Software}

Since our goal is to work on real Pioneer mobile robots developed by ActivMedia Robotics we have had to work with such robots in the Webots also. The Webots software already had the Pioneer robot in its package, but this robot could be used only for transport mobile robot class in Bionic Assembly System. For a assembly station robot we need a mobile manipulator which was obsolete in Webots. That was a reason to model a Pioneer robot with the arm on it. In this section we are presenting in details the modelling of robot arm for Pioneer mobile robot in Webots. Since the whole simulation is done with physics property it was a real challenge to simulate grasping and manipulating the objects taking in consideration the mass and friction factors of all objects. 


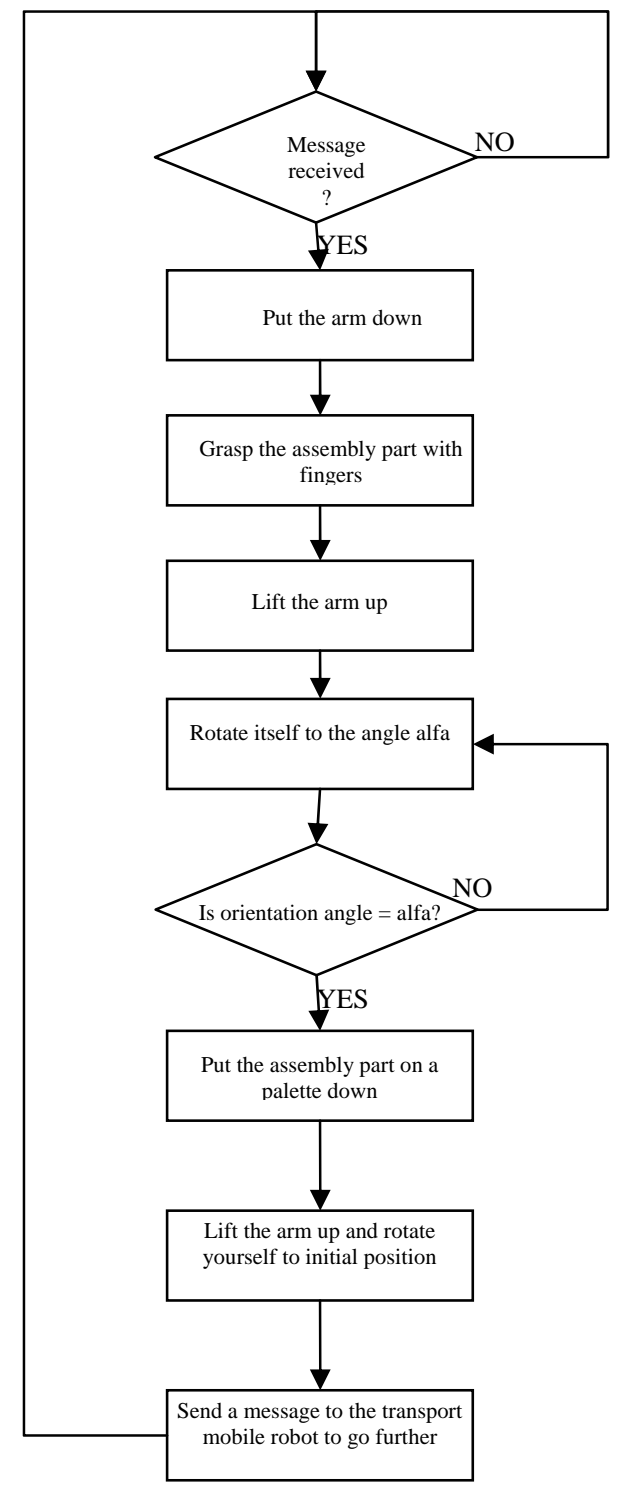

Figure 4: Controller of Assembly Station Robot.

On a Figure 5 (a, b, c, d) we can see the whole working cycle in simulation.

The elements of the arm are:

- Base - it serves as a joint between the arm and the robot. It is composed of cylinder and two cube objects.

- Lower arm - is a cylinder object, it has a servo motor on the bottom (on the joint with a base).

- Upper arm - is also a cylinder object equipped with a servo motor on a joint between it and the lower arm.

- Hand - is a cube object which serves as a base for fingers. The fingers are jointed with a hand.

- Fingers - jointed with a hand with a servo motor. They are composed of 2 elements finger and end of the finger. In that way arm is capable of grasping the cube and cylinder objects. 


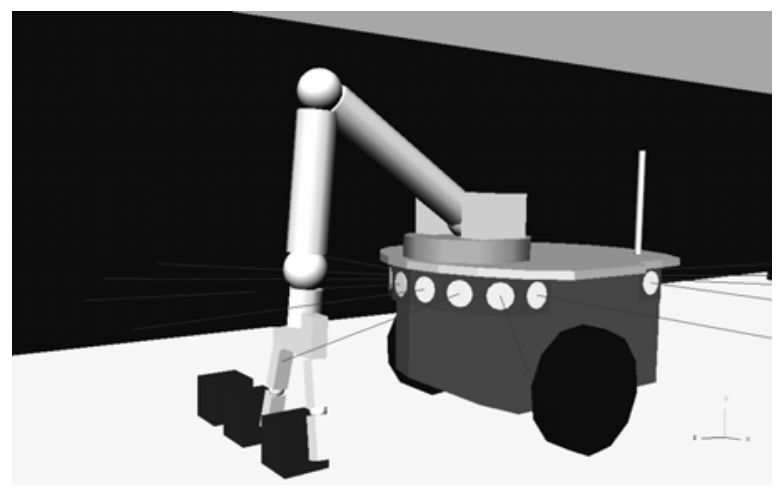

(a) grasping the assembly part

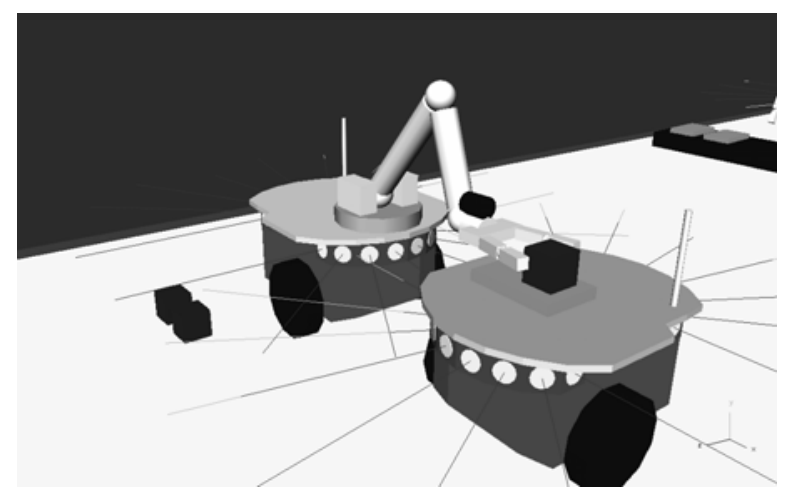

(c) leaving the part on the palette

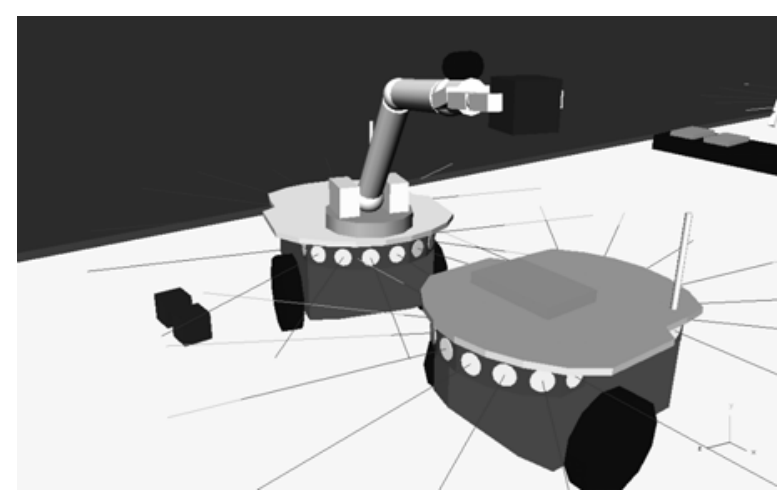

(b) position itself to a right angle

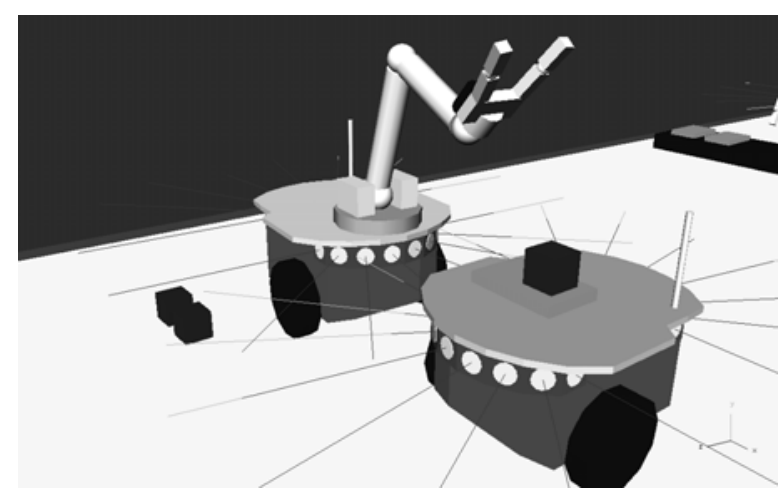

(d) lifting the arm up and returning to initial position

Figure 5: Assembly Station work cycle.

The parts with its motion and motion range are showed in table II.

Table II: Robot arm parts and allowance motion.

\begin{tabular}{|l|c|c|c|c|}
\hline \multicolumn{1}{|c|}{ Part } & Motion & \multicolumn{2}{c|}{$\begin{array}{c}\text { Dimensions } \\
\text { [m] }\end{array}$} & \multicolumn{2}{c|}{$\begin{array}{c}\text { Motion Range [deg.] } \\
\text { Min. } \\
\text { Max. }\end{array}$} \\
\hline Lower arm & Up and down & $\begin{array}{c}\text { height }=0.25 \\
\text { radius }=0.025\end{array}$ & -1.96 & 2.14 \\
\hline Upper arm & Up and down & $\begin{array}{c}\text { height }=0.17 \\
\text { radius }=0.025\end{array}$ & -2.07 & 2.07 \\
\hline Left Finger & $\begin{array}{c}\text { Left and } \\
\text { right }\end{array}$ & $\begin{array}{c}\mathrm{X}=0.055 \\
\mathrm{Y}=0.02 \\
\mathrm{Z}=0.02\end{array}$ & 0.71 & 1.75 \\
\hline End of left finger & $\begin{array}{c}\text { Left and } \\
\text { right }\end{array}$ & $\begin{array}{c}\mathrm{X}=0.05 \\
\mathrm{Y}=0.02 \\
\mathrm{Z}=0.02\end{array}$ & 0.00 & 1.06 \\
\hline Right Finger & $\begin{array}{c}\text { Left and } \\
\text { right }\end{array}$ & $\begin{array}{c}\mathrm{X}=0.055 \\
\mathrm{Y}=0.02 \\
\mathrm{Z}=0.02\end{array}$ & 1.41 & 2.17 \\
\hline End of Right Finger & $\begin{array}{c}\text { Left and } \\
\text { right }\end{array}$ & $\begin{array}{c}\mathrm{X}=0.05 \\
\mathrm{Y}=0.02 \\
\mathrm{Z}=0.02\end{array}$ & -1.06 & 0.00 \\
\hline
\end{tabular}


The robot with its components is shown in Fig. 6.

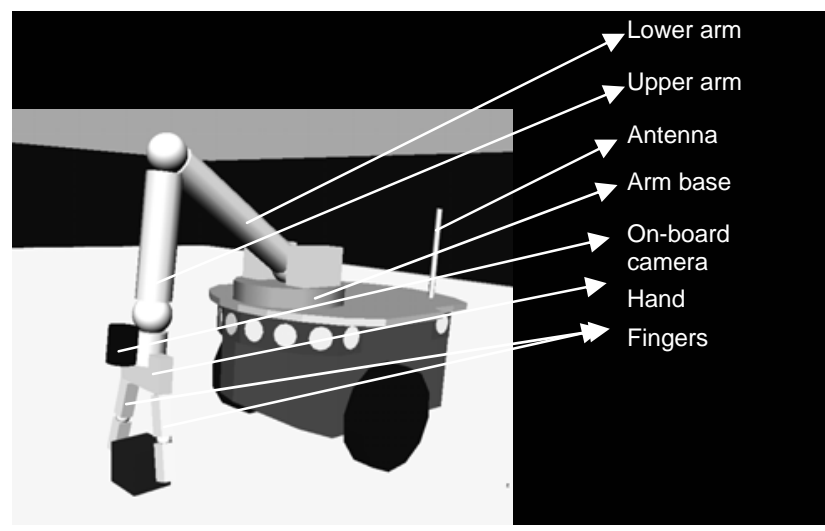

Figure 6: Assembly station robot with its components.

\section{WEBOTS SOFTWARE}

For simulation of Bionic Assembly System we have decided to use Webots professional software for simulation of mobile robots behaviour. This is the most realistic and reliable software of this kind at a moment. The simulation system used in Webots uses virtual time, making it possible to run simulations much faster than it would take on a real robot. Depending on the complexity of the setup and the power of your computer, simulations can run up to 300 times faster than the real robot when using the fast simulation mode. The graphical user interface of Webots allows you to easily interact with the simulation while it is running. The robot's behaviour is written using the $\mathrm{C}++$ or Java programming language. Moreover, any Webots controller can be connected to a third party software program, such as MatLab, LabView, etc. through a TCP/IP interface.

Once tested in simulation your robot controllers can be transferred to real robots. This is the real power of Webots, because there is no other software that has capability of transferring the controller programs on real robots.

More information about the software could be found in [9].

\section{CONCLUSION}

In this paper we have presented the Bionic Assembly System concept and in more details focused the paper on main robot classes: assembly robot station and transport mobile robot. Till now we have constructed a mobile arm in Webots software which can effectively manipulate cube and cylindrical objects. With the help of a vision system attached to the arm we can now start to develop a controller which will truly perform assembly tasks and not just pick and place mechanism. Regarding the transport mobile robot, we have showed that with a use of vector field histogram method, robots are able to navigate in multi-robot environment without collisions. The communication between the robots is solved using the radio emitter and receiver attached to each single robot.

The further work is to develop the controllers for both robot classes which will more deeply try to test the concept of Bionic Assembly System. So, the work presented in this paper is just a beginning in a realisation of a Bionic Assembly System. 


\section{REFERENCES}

[1] Parunak, van D. H. (1998). What can Agents do in industry, and why? An overview of industrially-oriented R\&D at CEC, Proceedings of CIA 98, USA

[2] Katalinic, B.; Kordic, V. (2002). Bionic Assembly System: Autonomous agents in selforganising complex flexible assembly system in CIM environment, The $4^{\text {th }}$ International Workshop on Emergent Synthesis - IWES 2002, Kobe, Japan

[3] Maeda, Y.; Kikuchi, H.; Izawa, H. (2003). An Easily Reconfigurable Robotic Assembly System, Proceedings of the 2003 IEEE International Conference on Robotics \& Automation, Taipei, Taiwan

[4] Asl, F. M.; Ulsoy, A. G.; Koren, Y. (2001) Dynamic Modeling and Stability of the Reconfiguration of Manufacturing Systems, Technical Report, University of Michigan

[5] Kordic, V.; Lazinica, A.; Merdan, M. (2005). Future of Manufacturing, International Journal of Advanced Robotic Systems, Vol. 2, No. 1, 071-081

[6] Lazinica, A.; Merdan, M.; Kordic, V. (2005) Navigation of Transport Mobile Robot in Bionic Assembly System, Proceedings of 2nd International Conference on Mechatronics - ICOM 2005, Kuala Lumpur, Malaysia, 100-106

[7] Borenstein, J.; Koren, Y. (1991). The Vector Field Histogram - A Fast ObstacleAvoidance for Mobile Robots, IEEE Journal of Robotics and Automation, Vol. 7, No. 3., 278-288

[8] Latombe, J. C. (1991). Robot Motion Planning, Kluwer Academic Publishers, Boston, MA, USA

[9] Michel, O. (2004). Cyberbotics Ltd - WebotsTM: Professional mobile robot simulation, International Journal of Advanced Robotic Systems, Vol. 1, No. 1, 40-43 